\title{
Sentido de vida: compreendendo este desafiador campo de estudo
}

\author{
Grazielli Padilha Vieira* (D) \\ Ana Cristina Garcia Dias
}

Universidade Federal do Rio Grande do Sul. Porto Alegre, RS, Brasil

\begin{abstract}
Resumo: Sentido de vida é uma variável que tem sido considerada central para compreensão do bem-estar humano nas últimas décadas. Entretanto, o estudo desse conceito é muito mais antigo e ele já recebeu contribuições da filosofia, da psicologia humanista, da logoterapia e, recentemente, da psicologia positiva e da psicometria, o que possibilitou que tal construto fosse mensurado. Este artigo explora os caminhos de sentido de vida enquanto construto ao longo do tempo, discutindo as contribuições que o conceito recebeu de diversas teorias psicológicas, incluindo o recente modelo tripartite de sentido de vida. Por fim, são discutidos os desafios enfrentados por pesquisadores e clínicos que buscam trabalhar com essa variável.
\end{abstract}

Palavras-chave: sentido de vida, psicologia positiva, logoterapia, psicometria.

\section{Introdução}

Sentido de vida (SV) tem sido considerado o núcleo e a chave para o entendimento do bem-estar, da motivação e uma necessidade humana (Frankl, 1946/2012; Heintzelman \& King, 2019, 2014; Hill, 2018). Segundo Seligman (2019), um dos fundadores do movimento da psicologia positiva, $\mathrm{SV}$ deve ser incorporado às pesquisas e práticas em psicologia para que estas sejam capazes de proporcionar às pessoas uma vida mais significativa, prazerosa, feliz e, portanto, que vale a pena ser vivida. Assim, esse conceito, ancorado diretamente na filosofia e discutido amplamente dentro da psicologia por Viktor E. Frankl (1946/2012), tem recebido cada vez mais espaço e reconhecimento dentro da pesquisa em psicologia. $\mathrm{O}$ conceito de SV adquiriu maior complexidade teórica e metodológica ao longo do tempo, sendo estudado desde as perspectivas mais empíricas até as mais filosóficoexistenciais (Thir \& Batthyány, 2016).

Faltam estudos teóricos que tratem dos caminhos do conceito de SV no cenário brasileiro. Por ser um conceito complexo, é necessário, ao pesquisador e ao clínico, conhecê-lo melhor para utilizá-lo em seu cotidiano. Assim, o objetivo deste artigo é apresentar as diferentes concepções teóricas e propostas empíricas sobre SV. Ainda, são descritos os modelos tradicionais e multidimensionais que teorizam sobre SV, apresentando-se o Modelo Tripartite de Sentido de Vida (MTSV) (George \& Park, 2016; Martela \& Steger, 2016), que tem possibilitado avanços interessantes à pesquisa empírica em SV, como a construção de escalas para a avaliação de SV enquanto variável indicativa

*Endereço para correspondência: graziellipadilhavieira@gmail.com de bem-estar. Por fim, são analisados os desafios de trabalhar com SV enquanto construto teórico mensurável, considerando as importantes contribuições do movimento da psicologia positiva a tais desafios.

\section{Da filosofia aos modelos multidimensionais de SV}

O ser humano busca encontrar sentido em sua existência desde os tempos antigos. A filosofia, a arte, a literatura, a matemática, a religião, entre outros ramos do conhecimento, dedicaram-se a compreender o sentido da existência humana. Essas áreas tratam do significado dessa existência de maneira ampla e filosófica, atribuindo um valor à vida como um todo, à experiência de estar vivo. Para compreender melhor como o SV surge enquanto temática da psicologia moderna, é preciso retomar o pensamento filosófico grego, que exerce enorme influência sobre o pensamento ocidental nessa temática.

$\mathrm{Na}$ Grécia antiga, Aristóteles discutiu amplamente o sentido da vida em uma perspectiva de entendimento da vida cotidiana, especialmente em sua obra Ética a Nicômaco (Aristóteles, 300 a.C./ 2016). O autor explora dois tipos de ética/condutas de vida: hedônica e eudemônica, sendo a primeira aquela que busca prazer e plenitude, em uma lógica mais individualista, e a última a que procura uma vida que vale a pena ser vivida, dotada de um valor transcendente. Esta seria a vida feliz, que só pode ser experimentada em comunidade, na conexão do sujeito com a pólis e com a cultura (Aristóteles, 300 a.C./ 2016).

A vida eudemônica agrega a necessidade dos indivíduos estarem conectados com suas comunidades, ligados a questões que vão além dos prazeres individuais. Desse modo, vincula a transcendência à participação na 
construção do bem comum, tornando-a mais concreta (Aristóteles, 300 a.C./2016). Essa é, portanto, a vida concreta, que pode ser vivida por qualquer pessoa, independentemente de sua classe social ou status, de seu lugar no mundo ou conhecimento filosófico. Aristóteles propõe, então, que a vida concreta e cotidiana do homem é dotada de significado, o que gerou uma nova perspectiva para pensar o homem real, contribuindo para que a vida com sentido se tornasse filosoficamente acessível a qualquer pessoa.

Essas ideias permaneceram restritas ao campo da filosofia até o início do século XX, quando a humanidade vivencia os episódios inéditos da Primeira e da Segunda Guerra Mundial e o sentido de tais acontecimentos na vida de cada pessoa passou a ser questionado. Assim, foram retomadas discussões sobre a constituição do ser humano, especialmente por Maslow e Sartre. Para Maslow (1962/2011), o ser humano é dotado de uma natureza interna, uma essência biologicamente alicerçada, que pode ser cientificamente estudada, sendo o sentido da vida definido a priori, pela própria condição humana. Sartre (1946/2014), por sua vez, defende que a existência é capaz de preceder a essência; sendo assim, o homem é livre e não possui uma natureza humana que o defina a priori. O sentido da vida precisa, então, ser construído ao longo da existência humana.

Porém, com os estudos de Viktor E. Frankl, um sobrevivente dos campos de concentração nazistas, a psicologia passa a se interessar verdadeiramente pelo SV. Frankl (1905-1997) foi um psiquiatra e psicólogo vienense que durante sua experiência nos campos buscou refletir sobre a experiência de estar e mantar-se vivo. Em 1945, após sua libertação, escreveu $O$ homem em busca de sentido (1946), livro no qual teorizou sobre SV de forma concreta. O autor trata SV como a vivência de um sentido individual calcado na singularidade de cada experiência humana (Frankl, 2012/1946).

A partir de suas observações nos campos de concentração, o autor descreve as diferenças entre os prisioneiros que não conseguiram lutar por suas vidas, os que seguiram adiante em busca de um futuro e os que conseguiram, inclusive, subverter a lógica de suas experiências nos campos de concentração. Frankl observa que os prisioneiros capazes de seguir em frente apesar da experiência vivida nos campos de concentração eram capazes de encontrar uma orientação futura para si e atribuir um valor significativo às relações afetivas fora do campo de concentração (filhos, amigos, cônjuges etc.). Assim, aqueles que conseguiram encontrar algum propósito em sua existência presente e futura empenhavam-se com afinco em sobreviver.

Para Frankl (1946/2012), o SV pode ser encontrado de diversas maneiras, especialmente por meio de três experiências concretas: (1) valores de criação: encontrar um trabalho ou atividade que exerça um papel significativo para o mundo; (2) valores de experiência: experimentar algo que possibilite a autotranscendência (exemplo: bondade, compaixão, amor etc.); ou (3) valores de atitude: escolher as atitudes diante do sofrimento inevitável e, se necessário, modificar aspectos pessoais para enfrentar o momento. Logo, SV pode variar ao longo da existência, conectando-se a diferentes objetos, metas ou experiências em diferentes momentos da vida, sendo um elemento central na constituição da saúde física e mental dos indivíduos.

Frankl não se dedicou somente à operacionalização do conceito de SV, mas também a sua utilização enquanto ferramenta terapêutica na logoterapia. Inicialmente foram desenvolvidos estudos de operacionalização de SV que entendiam o construto como unidimensional (Adler, 1940; Maddi, 1970), mas sem resultados satisfatórios. Assim, surgem os modelos multidimensionais de SV como alternativa que visava dar conta da complexidade do conceito e garantir seu caráter dinâmico (Greenberg \& Arndt, 2012; Greenberg, Pyszczynski, \& Solomon, 1986; Reker \& Wong, 1988). Entretanto, essa ampla literatura em torno de SV tornou a definição do próprio conceito ambígua. Cada proposta teórica definiu SV de maneira a condizer com suas expectativas e favorecer a mensuração do construto (Leontiev, 2013b; Steger, 2012). Na seção a seguir, apresentamos algumas dessas definições.

\section{Teorias em sentido de vida}

A literatura sugere que modelos multidimensionais são mais apropriados para a compreensão de construtos mais dinâmicos, como SV (Leontiev, 2013a). Esses modelos possibilitam avaliar SV enquanto um estado passível de mudança ao longo do tempo. SV não poderia ser mensurado enquanto um traço de personalidade, pois este pressupõe algo mais estável ao longo do tempo e cuja variação tende a ser mínima (George \& Park, 2017; Leontiev, 2013a, 2013b; Martela \& Steger, 2016). A partir das décadas de 1980 e 1990, surgem pesquisas que buscam entender a relação entre SV e outras variáveis, culminando em outros modelos de entendimento sobre SV, como o meaning making model (Park \& Folkman, 1997; Park, 2013), o meaning maintenance model (Heine, Proulx, \& Vohs, 2006; Proulx, 2013), o search or presence for meaning (Steger, Frazier, Oishi, \& Kaler, 2006), o terror management theory (Greenberg et al., 1986; Greenberg \& Arndt, 2012), e o MTSV (George \& Park, 2016; Martela \& Steger, 2016). Esses modelos serão melhor apresentados a seguir.

\section{Construção de sentido de vida (meaning making model)}

O modelo de construção de sentido de vida (Park \& Folkman, 1997; Park, 2010, 2013) trata das ferramentas que as pessoas utilizam para construir/ estruturar SV diante de situações estressantes capazes de provocar a quebra da coerência e do sentido em suas vidas, não propondo uma nova definição para o conceito. 
O modelo é composto por duas grandes dimensões: global meaning e situational meaning. A primeira dimensão envolve as crenças globais (global beliefs), os objetivos globais (global goals) e as ideias subjetivas de sentido (subjective sense of meaning). Ou seja, global meaning é composto pelo senso geral de orientação de um indivíduo no mundo. Já a dimensão situational meaning trata do SV em uma situação concreta no ambiente, considerando a interpretação que o indivíduo faz desta, e se inicia a partir de uma situação estressante ou considerada incoerente (Park, 2010, 2013).

Esse modelo teórico não deixa clara a definição do que seria estressor, dependendo da avaliação subjetiva de cada indivíduo para que uma situação seja classificada como estressante ou não. A partir dessa avaliação, inicia-se o processo de construção de um sentido para uma nova experiência, como forma de lidar com as incoerências do ambiente em relação às crenças do indivíduo (Park, 2010).

Esse processo pode ser feito mediante diversos recursos, tanto automáticos, quanto cognitivos e emocionais. Podem ser desenvolvidos processos de assimilação e de busca por compreensão do estressor, sendo a construção de novos sentidos (meanings made) o resultado desse trabalho psíquico. Alguns produtos desse processo podem ser a ideia de que a vida tem sentido, aceitação, entendimento causal, percepção de crescimento e/ou mudanças de vida positivas, mudanças de crenças globais e objetivos, restauração e/ou mudança no SV, entre outros (Park, 2010).

Esse modelo tem auxiliado pesquisadores a compreender os processos de construção e reconfiguração de SV diante da vivência de situações traumáticas incontroláveis, como o enfrentamento a doenças graves.

\section{Manutenção de sentido de vida (meaning maintenance model)}

O modelo de manutenção de sentido de vida proposto por Heine et al. (2006) pressupõe que as pessoas constroem o sentido de sua existência a partir de suas expectativas e aprendizados sobre as relações estabelecidas com o ambiente, ou seja, o mundo tem sentido na medida em que atende às expectativas/crenças que o indivíduo nutre sobre ele (Heine et al., 2006; Proulx, 2013; Proulx \& Inzlicht, 2012). Nesse modelo, por exemplo, uma pessoa que acredita fortemente que $o$ mundo é um lugar justo, ao se deparar com uma possível injustiça, precisa revisar tanto suas expectativas como suas aprendizagens sobre o mundo, e logo precisa rever também seu SV.

Portanto, é preciso compreender como os indivíduos lidam com a quebra das expectativas, sejam elas positivas ou negativas. A teoria postula que existem cinco maneiras cognitivas de lidar com as quebras de expectativas: assimilação, acomodação, afirmação, abstração e construção (assembly) de um novo SV. Essas estratégias exigem esforço cognitivo e afetivo dos indivíduos. As quebras de expectativas podem ocorrer por meio de eventos traumáticos ou de mudanças sutis, como estudos empíricos têm revelado (Dechesne \& Kruglanski, 2004; Proulx \& Heine, 2008; Proulx \& Inzlicht, 2012).

A partir desse modelo, surge a possibilidade de considerar a interpretação das pessoas sobre o mundo e sobre suas relações como um componente fundamental para a construção de SV. Portanto, passa a ser relevante o senso de coerência e a compreensão que as pessoas têm sobre o mundo (George \& Park, 2016).

\section{Teoria de gerenciamento do terror (terror management theory)}

A teoria de gerenciamento do terror não é uma teoria sobre SV propriamente, entretanto trata da maneira como as pessoas lidam com a consciência constante e iminente da própria morte, contribuindo para a compreensão de SV (Greenberg et al., 1986). A simples consciência da morte geraria um estado constante de terror que necessitaria ser manejado ao longo do tempo. Para lidar com isso, os indivíduos devem criar a sensação de pertencimento a uma família, comunidade ou cultura que possibilite balancear o terror gerado pela expectativa da finitude, desenvolvendo um valor associado a estar vivo, em contato com um mundo significativo, em busca de um sentido para a existência (Greenberg et al., 1986; Solomon, Greenberg, \& Pyszczynski, 1991).

A ideia de que a vida é dotada de valor permite a construção da autoestima, definida como a percepção dos indivíduos sobre sua capacidade de contribuir para a construção de sentido (Greenberg \& Arndt, 2012). Dessa forma, as pessoas agem no mundo visando evitar a própria morte, na tentativa de assegurar a imortalidade, de forma simbólica ou concreta. A transcendência proporciona aos indivíduos alcançar certa imortalidade simbólica através do papel ativo que têm no mundo, na construção do sentido da existência (Greenberg et al., 1986; Greenberg, Solomon, \& Arndt, 2008; Solomon et al., 1991). Ou seja, os indivíduos precisam viver a sensação de que suas vidas e escolhas repercutem além deles mesmos, como uma forma de manterem-se conectados e vivos por meio da criação do mundo e da sociedade que os cerca, garantindo sua imortalidade através da cultura e do senso de pertença (George \& Park, 2016, 2017; Greenberg \& Arndt, 2012).

\section{Presença e busca por sentido (presence and search for meaning)}

Esse modelo avalia SV a partir de uma abordagem mais relativista ante os recursos que os indivíduos utilizam para a construção de SV. São propostas duas dimensões para a compreensão do SV: a busca por SV e a presença de SV. A primeira trata da motivação ou direção que os indivíduos desenvolvem para obter SV. Já a segunda é sobre compreender e identificar se os indivíduos apresentam um claro e definido SV (Steger et al., 2006). A partir dessa 
concepção foi desenvolvida uma escala do tipo Likert, composta por dez itens, sendo cinco relativos à dimensão de busca por SV e cinco à dimensão de presença de SV (Steger et al., 2006). Assim, um indivíduo que apresenta alta busca de sentido, tenderia, teoricamente, a apresentar baixa presença, bem como o indivíduo que apresenta uma alta presença, tenderia a apresentar pontuações baixas em busca de sentido (Steger et al., 2006).

Os itens que compõem a escala, criados por uma equipe de pesquisadores a partir de uma definição prévia do que seria SV, utilizaram o critério inicial de validade aparente. Após isso, eles foram testados em uma amostra e realizou-se a análise confirmatória dos dados para decidir quais deles eram mais adequados e comporiam a escala final. Assim, a escala ganhou destaque por se propor a avaliar SV como um construto mensurável quantitativamente. Ela tem sido traduzida, adaptada e utilizada em diversos países, obtendo indicadores satisfatórios de qualidade psicométrica (Aquino et al., 2015; Damásio \& Koller, 2015; Steger et al., 2006).

Entretanto, por utilizar uma base teórica mais relativista, sem abordar necessariamente os mecanismos psíquicos, cognitivos, culturais ou motivacionais envolvidos nos processos de busca e presença de sentido, esse modelo teórico encontrou certa dificuldade em explicar possíveis variações culturais em SV. Por exemplo, no estudo de Steger, Kawabata, Shimai e Otake (2008) foram avaliados adultos jovens norteamericanos e japoneses em relação a busca e presença de SV. O estudo observou que, para os norte-americanos, quando a presença de sentido obtinha uma pontuação alta, a busca por sentido apresentava uma pontuação baixa, seguindo o previsto pelos autores. Entretanto, os jovens japoneses que apresentavam uma pontuação alta em busca também apresentavam uma alta pontuação em presença de sentido, contrariando as expectativas da teoria. Esse resultado sugere que busca e presença não são conceitos opostos em termos de SV, como proposto pela teoria, mas poderiam ser dimensões complementares em algumas culturas. Os autores concluem que a busca de sentido é influenciada pela cultura e também é capaz de moderar a influência cultural na presença de sentido (Steger et al., 2008).

Desse modo, o modelo tem dificuldade em oferecer maiores explicações sobre a diferença cultural encontrada entre norte-americanos e japoneses, apenas indicando que tal diferença existe. Faltam recursos teóricos e mesmo técnicos capazes de oferecer uma explicação clara e concisa sobre quais aspectos da construção de sentido ou mesmo da busca por sentido são diferentes nessas culturas. Destaca-se a importância do modelo, mesmo diante de suas limitações, pois foi por meio dele que o tópico de SV foi retomado pelo campo da mensuração. Hoje o próprio Steger (Martela \& Steger, 2016) tem abordado sentido de vida a partir do modelo multidimensional, propondo que se defina e se operacionalize o conceito por meio de recursos que as pessoas utilizam para construir seu SV.

\section{Modelo tripartite de sentido de vida}

Recentemente, Steger (2012) buscou construir uma definição de SV que sintetizasse aspectos presentes nos modelos teóricos anteriores, buscando superar a ambiguidade das definições propostas para SV. Para o autor,

Sentido é a rede de conexões, entendimentos e interpretações que nos auxilia a compreender nossa experiência e formular planos para direcionar nossas energias para alcançarmos o futuro que desejamos. Sentido nos oferece a percepção de que nossas vidas importam, são coerentes e que são formadas por mais do que a soma dos segundos, dias e anos. ${ }^{1}$ (p. 65)

George e Park (2016) também propuseram uma definição de SV multidimensional. Para os autores "SV é formado na medida em que vida do indivíduo é experimentada como fazendo sentido, sendo direcionada e motivada por objetivos válidos, e como relevantes para o mundo" (p. 206). A proposta do MTSV favorece uma maior integração teórica que, por sua vez, facilita a operacionalização do conceito e o desenvolvimento de instrumentos para mensuração de SV, como a Multidimensional Existential Meaning Scale (George \& Park, 2017) e a Multidimensional Meaning in Life Scale (Costin \& Vignoles, 2019). Isso ocorre porque os modelos reúnem evidências tanto das teorias focadas em estruturas de SV - por exemplo: meaning making model, meaning maintenance model - quanto das teorias em torno da experiência de SV - por exemplo: teoria de Reker e Wong (1988).

Os modelos de MTSV, propostos por Martela e Steger (2016) e George e Park (2016, 2017), apresentam as mesmas três dimensões para SV. São elas, respectivamente: compreensão (coherencelcomprehension), propósito (purpose) e valorização (significance/mattering). O MTSV foi formulado tanto a partir do quadro conceitual presente nas principais perspectivas teóricas de $\mathrm{SV}$, que vimos anteriormente, como de pesquisas empíricas sobre o tema (Frankl 1946/2012; George \& Park, 2016; Heine et al., 2006; Martela \& Steger, 2016; Park \& Folkman, 1997; Reker \& Wong, 1988; Steger et al., 2006).

A dimensão compreensão (comprehension/ coeherence) do MTSV se refere à sensação de que a existência é coerente e compreensível, permitindo a conexão entre as experiências vividas no passado e presente e a construção de uma noção de futuro, proporcionando certa linearidade a história de vida dos indivíduos. Refere-se, de certa forma, à compreensão dos próprios caminhos e direções que a vida toma, sendo necessária a busca por consistência e coerência entre o que se espera da vida e a realidade experimentada pelos indivíduos (George \& Park, 2016; Heine et al., 2006).

1 Livre tradução nossa.

2 Livre tradução nossa. 
Essa dimensão engloba os aspectos mais cognitivos do modelo de Reker e Wong (1988) e se aproxima do modelo de manutenção de SV desenvolvido por Heine et al. (2006) e Proulx \& Inzlicht (2012). Em indivíduos saudáveis ela contribui para que sejam minimizados sentimentos de incerteza e ansiedade, pois possibilita a adoção de estratégias mais funcionais ante situações ambíguas/inesperadas e uma melhor orientação nos caminhos da vida (George \& Park, 2016).

No estudo conduzido por Costin e Vignoles (2019) para a construção da escala de SV, a compreensão foi a dimensão que apresentou resultados mais incoerentes com as expectativas teóricas. Os autores propõem que o senso de compreensão pode ser antes um efeito da percepção de sentido na existência do que um componente formador da noção de SV. Isso suscita a necessidade de novos estudos sobre esse componente do MTSV, que possibilitem seu melhor desenvolvimento teórico e operacionalização.

Já a dimensão propósito (purpose) representa os aspectos mais motivacionais do modelo, referindo-se aos objetivos e direção que o indivíduo tem em sua vida e o quanto está comprometido com tais objetivos (Hill, 2018; Reker \& Wong, 1988; Shin \& Steger, 2014). Esse é um construto clássico e o que mais se aproxima da teoria original proposta por Viktor Frankl (1946/2012), que sugere que o SV está vinculado à identificação dos objetivos de vida da pessoa, à identificação de sua missão (Battista \& Almond, 1973; George \& Park, 2016; Mcknight \& Kashdan, 2009; Rabin, 1979). Propósito não diz respeito apenas a fazer coisas, mas também à capacidade humana de definir metas de curto, médio e longo prazo, articulando comportamentos e situações para alcançá-las. Esses objetivos, dotados de significado na construção da narrativa de vida, fornecem conexões entre passado, presente e futuro, por meio do estabelecimento de uma hierarquia entre metas (George \& Park, 2016; Leontiev, 2013a, 2013b).

O terceiro componente do MTSV denomina-se valorização (mattering/significance) e trata da atribuição de um valor inerente à própria vida (George \& Park, 2016, 2014; Martela \& Steger, 2016). É a percepção de que a existência está ligada a algo maior que o próprio indivíduo, como uma comunidade, cultura ou época e implica necessariamente a noção de transcendência. São incorporados os componentes afetivos da teoria de Reker e Wong (1988) e a teoria do gerenciamento de terror (Greenberg \& Arndt, 2012; Greenberg et al., 1986) ao MTSV.

A busca por valorização envolve, portanto, a tarefa de evitar a morte, transcender, e lidar com a ansiedade existencial, bem como a construção de relações interpessoais significativas (Greenberg \& Arendt, 2012; Reker \& Wong, 1988). A noção de transcendência contribui significativamente para a construção do valor de si mesmo, conectando o indivíduo com a sociedade e a cultura ao seu redor, inclusive na possibilidade de colaborar para a construção de ambas (George \& Park, 2016; Greenberg et al., 1986). A valorização tem sido pouco estudada enquanto construto isolado (George \& Park, 2014), sendo possível pensar que ela atua também como uma medida independente de bem-estar, indo além do papel de componente de SV (Damásio, Hauck-filho, \& Koller, 2014). Logo, é importante a realização de mais estudos nessa dimensão do MTSV, visando aperfeiçoar o entendimento da relação com SV, operacionalização do conceito e avaliação, especialmente com a população brasileira.

Essas dimensões que compõem o MTSV devem ser pensadas como fortemente inter-relacionadas, influenciando-se mútua e continuamente, em um modelo fatorial correlacionado (George \& Park, 2017; Martela \& Steger, 2016). A partir do MTSV, é proposta a Multidimensional Existential Meaning Scale (MEMS) (George \& Park, 2017), que busca mensurar as três dimensões do modelo, fornecendo escores para cada uma delas. Os itens da escala foram construídos a partir da definição teórica das dimensões. Foram desenvolvidos inicialmente 43 itens, avaliados por oito juízes especialistas na temática SV ou em avaliação psicológica.

$\mathrm{Na}$ versão final, composta por quinze itens, permaneceram apenas os julgados adequados estatisticamente, sendo incorporadas sugestões de novos itens propostos pelos avaliadores. A escala apresentou boa consistência interna e a capacidade de acessar individualmente cada dimensão, fornecendo dados para a averiguação de cada uma delas e das possíveis articulações entre as dimensões (George \& Park, 2017). A MEMS apresentou correlações positivas com variáveis, como bem-estar subjetivo, satisfação com a vida, espiritualidade/ religiosidade (George \& Park, 2017a), afeto positivo; e correlações negativas, como afeto negativo, depressão, ansiedade e estresse (George \& Park, 2017).

\section{Desafios a serem consideradas na avaliação de sentido de vida}

Leontiev (2013a), George e Park (2016) e Martela e Steger (2016) descrevem as limitações que pesquisadores encontram ao abordarem SV enquanto um construto psicológico mensurável. A primeira delas é o fato de que é difícil fazer uma leitura integrada da vasta literatura, empírica e teórica, sobre SV (George \& Park, 2016; Leontiev, 2013a): SV tem sido dividido em diversas áreas (estruturas de sentido, experiências de SV, intervenções em SV, estudos teóricos em SV) e cada pesquisador utiliza uma definição, o que implica diversos métodos de avaliação do mesmo construto e a utilização de medidas, por vezes, incongruentes entre um estudo e outro, o que dificulta comparações interculturais e entre estudos (George \& Park, 2016).

É preciso, ainda, lidar com as dificuldades operacionais de definição de SV, que têm se revelado um desafio para a integração da literatura em torno da temática. Alguns estudos avaliam SV a partir de recursos psíquicos que os indivíduos utilizam para sua estruturação (meaning framework), enquanto outros tratam dos julgamentos que 
os indivíduos fazem sobre a percepção de sentido em sua existência (meaning in life judments). Recentemente, mesmo algumas áreas mais comportamentalistas têm se aventurado a explorar a ideia de SV (Kanter, Busch, \& Rusch 2009) construindo outras possíveis definições do conceito.

Outra dificuldade é a tendência de considerar o conceito de forma unidimensional. Essa visão limita a compreensão de SV enquanto fenômeno complexo, que trata da experiência humana, vinculada a diferentes aspectos mutáveis da vida. Portanto, uma compreensão unidimensional traça um limite para o que pode ser entendido como uma existência dotada de sentido. Alguns autores consideram que a adoção de uma perspectiva multidimensional impossibilita que SV atue como uma variável que possibilita aos indivíduos transcenderem seus limites, alcançando novas possibilidades para sua existência (George \& Park, 2016; Leontiev, 2013a, 2013b; Martela \& Steger, 2016), tornando SV uma simples equação de causa e efeito.

Ainda, é preciso considerar a ambiguidade conceitual em torno da definição de SV. Para Leontiev (2013a) é preciso considerar que "Sentido é, portanto, uma construção hipotética, algo não diretamente observável, mas sim concebido. É necessário para explicar fenômenos observáveis, mas não pode ser reduzido a eles"3 (p. 463). Ou seja, Leontiev propõe uma visão mais existencialista do conceito. Para o autor, SV é uma experiência, uma maneira cognitiva e emocional de interpretar o mundo, um fenômeno que atravessa a experiência humana, estando associado à motivação, compreensão da própria história de vida e consideração de si mesmo para o mundo em uma perspectiva transcendente.

SV também se correlaciona a outras variáveis, como afeto positivo, afeto negativo, felicidade, ansiedade e depressão, que são fortemente vinculadas às experiências cotidianas de ser e estar no mundo e, por vezes, pode ser confundido com elas pelos participantes das pesquisas. O autor sugere que, por conta dessa confusão, é possível que, ao avaliarmos SV sem a necessária preocupação em se fazer entender pelos participantes, há o risco de estarmos avaliando, na verdade, outros construtos (Leontiev, 2013a, 2013b).

Para esses autores de cunho mais existencialista, SV não pode ser reduzido a uma medida/variável (Hill, 2018; Leontiev, 2013b). Dessa forma, SV enquanto experiência nunca pode ser inteiramente compreendido ou inteiramente mensurado, sendo esse um aspecto fundamental do próprio conceito (Frankl, 1946/2012; Leontiev, 2013b). Isso sugere que viver uma vida dotada de sentido envolve uma gama de sentimentos e comportamentos que permeiam o cotidiano da vida significativa. Esses comportamentos e sentimentos podem ser avaliados, apresentando indicadores de SV e recursos cognitivos, afetivos e culturais presentes nessa experiência (Hill, 2018; Leontiev, 2013b). Assim, é possível avaliar apenas algumas projeções de SV presentes

3 Livre tradução nossa. no comportamento, cognição, cultura e interação dos sujeitos com o mundo ao seu redor e inferir a partir disso uma "medida/quantificação" de SV. Contudo, há de se reconhecer a complexidade do conceito e que as facetas que estão sendo mensuradas podem não corresponder a sua totalidade.

Há também a questão semântica. A palavra "sentido" apresenta possibilidades diversas de significado na linguagem coloquial, que implicam diretamente o entendimento dos participantes de pesquisas sobre SV e no desenvolvimento teórico dos estudos (Leontiev, 2013a). Uma dessas possibilidades é a ideia de direção, sentido em que dado objeto está indo. A dimensão de propósito do MTSV traz a ideia da direção que a existência do indivíduo está tomando como parte desse aspecto motivacional. Outra definição possível na linguagem coloquial de sentido implica a noção de conexões, encadeamento entre pessoas, objetos ou ideias. Essa ideia se aproxima da dimensão de valorização, na qual tais conexões são fundamentais para a atribuição de valor à própria existência (Baumeister \& Vohs, 2002; Proulx \& Inzlicht, 2012).

Sentido, enquanto senso, também carrega a noção de percepção ou compreensão da própria história e dos caminhos traçados para a vida. Dessa forma, também se aproxima da dimensão de compreensão proposta no MTSV. Assim, para perceber sentido na vida, cabe considerar o que se pode compreender, narrar e contar dessa vida. O inenarrável, aquilo que é da ordem do incoerente e do inexplicável, tende a diminuir a percepção dos indivíduos de SV (Hill, 2018; George \& Park, 2016). Já a noção de sentido enquanto ponto de vista, modo de considerar, aspecto ou face de algo, remete à questão da diversidade e dos aspectos culturais envolvidos ao se estudar SV e abre espaço para se refletir sobre como SV pode ser uma experiência diferente em cada parte do mundo, em cada cultura. Cabe considerar a cultura em sua definição de rede de compartilhamento de sentidos e expectativas, produzida, distribuída e modificada por um grupo de indivíduos interconectados enquanto uma grande fonte de SV (Chiu \& Hong, 2007).

Ainda sobre a cultura, é preciso considerar sua capacidade de conectar as pessoas com o transcendente, pois ela liga os indivíduos a algo maior que eles mesmos, como as tradições, hábitos e aspectos históricos de constituição de um povo. A cultura é também um recurso para a construção do valor de si. Dessa forma, é capaz de oferecer esperança ao dar às pessoas a possibilidade de conceber o mundo e a existência como algo transcendente e duradouro (Chao \& Kesebir, 2013).

Portanto, SV é uma variável com forte influência dos aspectos culturais e as variações culturais afetam diretamente o entendimento e a operacionalização das dimensões de SV. Como vimos anteriormente, o estudo conduzido por Steger et al. (2008) comparou índices de busca e presença de SV entre estudantes norte-americanos e japoneses para avaliar os aspectos culturais envolvidos no construto. Esse trabalho demonstra como uma cultura é capaz de disseminar um modo de relacionar-se com o 
mundo e com a vida, modificando diretamente os índices que os sujeitos apresentam em medidas como o SV. Ainda, ilustra a necessidade de cuidados a serem adotados na adaptação ou desenvolvimento de instrumentos sobre um dado constructo, uma vez que a cultura apresenta concepções e práticas que precisam ser levados em consideração (Chao \& Kesebir, 2013; Steger et al., 2008).

Destacamos que os indivíduos nem sempre estão familiarizados com a questão do sentido de suas vidas e isso faz com que seu entendimento sobre SV possa variar amplamente (Leontiev, 2013a). De modo geral, a maioria das pessoas não tem clareza sobre o que está sendo perguntado ao deparar-se com uma pesquisa sobre $\mathrm{SV}$, diferentemente do que ocorre com outras variáveis psicológicas mais estudadas. Isso pode gerar confusão com outras variáveis psicológicas. Assim, ao tentar avaliar SV podemos estar, na verdade, avaliando uma de suas muitas projeções relacionadas ao construto e não SV propriamente (Chao \& Kasebir, 2013; Leontiev, 2013a). SV trata de uma percepção global da experiência de vida, tornando-se algo muito complexo e menos palpável para os participantes dos estudos, uma vez que não diz respeito apenas a um único momento da existência, como as pessoas tendem a entender outras variáveis (Leontiev, 2013a).

\section{Considerações finais}

As complexidades que envolvem o entendimento de SV enquanto conceito e variável, bem como sua longa história na filosofia e, recentemente, na psicologia, tornam o conceito bastante distante da realidade dos participantes dos estudos e do cotidiano dos pesquisadores. No caso desses últimos, existem poucas pesquisas brasileiras que deem conta da complexidade de SV enquanto construto ou mesmo que o incluam entre suas variáveis investigadas ou que se dediquem ao desenvolvimento conceitual, teórico e prático de SV.
SV é um construto de grande relevância para a saúde mental das mais variadas populações e culturas. Mesmo assim, o estudo e a mensuração de SV enquanto construto psicológico ainda se mostram desafiadores ao pesquisador. É preciso considerar questões culturais, semânticas e mesmo de clareza conceitual do construto para garantir uma pesquisa de qualidade. Ainda, SV mostra-se um construto relevante à psicologia à medida que se percebe com mais clareza seu papel fundamental para o desenvolvimento saudável dos indivíduos, especialmente por meio de suas conexões com outras variáveis mais conhecidas.

Orecente MTSV traz uma proposta multidimensional mais robusta para o entendimento de SV; entretanto, é necessário aprofundar os estudos empíricos e teóricos em suas dimensões, principalmente as dimensões de valorização e compreensão. Mesmo diante do adequado funcionamento das escalas que seguem o MTSV no cenário internacional, o modelo e os instrumentos desenvolvidos a partir dele ainda carecem de evidências de validade para o contexto brasileiro.

Por fim, as futuras pesquisas na temática devem atentar-se aos diferenciais culturais e à profundidade teórica necessária para uma boa utilização do conceito e do construto, bem como à construção de instrumentos de mensuração. Cabe destacar a necessidade de estudos que envolvam programas de intervenção e protocolos voltados a construção de SV, hoje inexistentes no cenário brasileiro. Fora do país existem algumas propostas bastante interessantes, voltadas diretamente à construção e ao aumento da experiência de SV (Shin \& Steger, 2014). Observa-se também a necessidade de novos estudos no cenário brasileiro considerando as diversas mudanças recentes pelas quais o construto tem passado, especialmente nos últimos dez anos. É preciso compreender quais influências culturais e sociais atravessam a construção da ideia de SV para os brasileiros de maneira mais profunda.

\section{Meaning in life: understanding this challenging field of study}

Abstract: Meaning in life has been considered a key variable to the understanding of human well-being in the last decades. However, the study of this concept is much older. Meaning in life received contributions from Philosophy, Logotherapy, and recently from Positive Psychology and psychometric studies, thus becoming measurable. This article explores the paths of meaning in life as a construct over time and discuss the contributions of some psychology theories to the concept, including the Tripartite View of Meaning in Life. Finally, we discuss the challenges faced by researchers and clinical psychologists to work with this construct.

Keywords: meaning in life, positive psychology, logotherapy, psychometric measures.

\section{Sens de la vie : comprendre ce domaine d'étude difficile}

Résumé : Le sens de la vie est une variable qui a été considérée comme essentielle pour comprendre le bien-être humain au cours des dernières décennies. Cependant, son étude est beaucoup plus ancienne, en ayant reçu des contributions de la philosophie, de la psychologie humaniste, de la logothérapie et récemment, de la psychologie positive et de la psychométrie, rendant ce concept mesurable. Cet article discute les voies de sens de la vie en tant que concept construit au fil du temps, 
en examinant les contributions qu'il a reçues de diverses théories psychologiques, y compris le récent modèle tripartite de sens de la vie. Enfin, on discute les défis auxquels sont confrontés les chercheurs et les cliniciens qui cherchent à travailler avec cette variable.

Mots-clés : sens de la vie, psychologie positive, logothérapie, psychométrie.

\section{Sentido de la vida: comprender este desafiante campo de estudio}

Resumen: El sentido de la vida es una variable que se ha considerado fundamental para comprender el bienestar humano en las últimas décadas. Sin embargo, el estudio de este concepto es mucho más antiguo y ya ha recibido aportes de la filosofía, la psicología humanística, la logoterapia y, recientemente, de la psicología positiva y la psicometría, haciéndolo medible. Este artículo explora las trayectorias del sentido de la vida como un constructo a lo largo del tiempo, discutiendo las contribuciones que el concepto ha recibido de varias teorías psicológicas, incluido el reciente modelo tripartito de sentido de la vida. Finalmente, se discuten los desafíos que enfrentan los investigadores y clínicos que buscan trabajar con esta variable.

Palabras clave: sentido de la vida, psicología positiva, logoterapia, psicometría.

\section{Referências}

Adler, A. (1940). A ciência de viver. Rio de Janieiro, RJ: José Olympio.

Aquino, T. A. A., Veloso, V. G., Aguiar, A. A., Serafim, T. D. B., Pontes, A. M., Pereira, G. A., \& Fernandes, A. S. (2015). Questionário de sentido de vida: Evidências de sua validade fatorial e consistência interna. Psicologia: Ciência e Profissão, 35(1), 4-19. doi: 10.1590/1982-3703001332012

Aristóteles. (2016). Ética a Nicômaco (L. F. de Souza, trad.). São Paulo, SP: Martin Claret. (Trabalho original publicado em 300 a.C.).

Battista, J., \& Almond, R. (1973). The Development of Meaning in Life. Psychiatry, 36, 409-427. doi: 10.1080/00332747.1973.11023774

Baumeister, R. F., \& Vohs, K. D. (2002). The pursuit of meaningfulness in life. In C. R. Snyder \& S. J. Lopez (Eds.), Handbook of positive psychology (pp. 608-618). Oxford: Oxford University Press.

Chao, M. M., \& Kesebir, P. (2013). Culture: The grand web of meaning. In J. A. Hicks \& C. Routledge (Orgs.), The experience of meaning in life: Classical perspectives, emerging themes, and controversies (pp. 317-331). Dordrecht: Springer. doi: 10.1007/978-94-007-6527-6_24

Chiu, C., \& Hong, Y. (2007). Cultural processes: Basic principles. In A. W. Kruglanski \& E. T. Higgins (Eds.), Social psychology: Handbook of basic principles (pp. 785-804). New York: Guilford Press.

Costin, V., \& Vignoles, V. L. (2019). Meaning is about mattering: Evaluating coherence, purpose, and existential mattering as precursors of meaning in life judgments. Journal of Personality and Social Psychology, 118(4), 864-884. doi: $10.1037 /$ pspp0000225

Damásio, B. F., Hauck-Filho, N., \& Koller, S. H. (2016). Measuring meaning in life: An empirical comparison of two well-known measures. Journal of Happiness Studies, 17(1), 431-445. doi: 10.1007/s10902-014-9602-8
Damásio,B., \&Koller,S.(2015). Meaning in LifeQuestionnaire: Adaptation process and psychometric properties of the Brazilian version. Revista Latinoamericana de Psicología, 47(3), 185-195. doi: 10.1016/j.rlp.2015.06.004

Dechesne, M., \& Kruglanski, A. W. (2004). Terror's epistemic consequences: Existential threat and the quest for certainty and closure. In J. Greenberg, S. L. Koole, \& T. Pyszczynski (Eds.), Handbook of Experimental Existential Psychology (pp. 247-262). New York: Guilford Press.

Frankl, V. (2012). O homem em busca de um sentido (F. J. Gonçalves, trad.). Alfragide: Lua de Papel. (Trabalho original publicado em 1946).

George, L. S., \& Park, C. L. (2014). Existential mattering: Bringing attention to a neglected but central aspect of meaning? In A. Batthyany \& P. Russo-Netzer (Eds.), Meaning in positive and existential psychology (pp. 39-51). New York: Springer. doi: 10.1007/978-1-4939-0308-5_3

George, L. S., \& Park, C. L. (2016). Meaning in life as comprehension, purpose, and mattering: Toward integration and new research questions. Review of General Psychology, 20(3), 205-220. doi: 10.1037/gpr0000077

George, L. S., \& Park, C. L. (2017). The Multidimensional existential meaning scale: A tripartite approach to measuring meaning in life.Journalof PositivePsychology, 12(6), 613-627. doi: 10.1080/17439760.2016.1209546

Greenberg, J., \& Arndt, J. (2012). Terror management theory. In P. A. M. Van Lange, A. W. Kruglanski \& E. T. Higgins (Eds.), Handbook of theories of social psychology (pp. 398-415). Thousand Oaks: Sage. doi: 10.4135/9781446249215.n20

Greenberg, J., Pyszczynski, T., \& Solomon, S. (1986). The causes and consequences of a need for self-esteem: A terror management theory. In R. F. Baumeister (Org.), Public self and private self (pp. 189-212). New York: Springer. doi: 10.1007/978-1-4613-9564-5_10 
Greenberg, J., Solomon, S., \& Arndt, J. (2008). A basic but uniquely human motivation: Terror management. In J. Y. Shah \& W. L. Gardner (Eds.), Handbook of motivation science (pp. 114-134). New York: Guilford Press.

Heine, S. J., Proulx, T., \& Vohs, K. D. (2006). The meaning maintenance model: On the coherence of social motivations. Personality and Social Psychology Review, 10(2), 88-110. doi: 10.1207/s15327957pspr1002_1

Heintzelman, S. J., \& King, L. A. (2014). Life is pretty meaningful. American Psychologist, 69(6), 561-574. doi: $10.1037 / \mathrm{a} 0035049$

Heintzelman, S. J., \& King, L.A. (2019). Routinesand meaning in life. Personality and social psychology bulletin, 45(5), 688-699. doi: 10.1177/0146167218795133

Hill, C. E. (2018). Meaning in life: A therapist's guide. Washington: American Psychological Association. doi: 10.1037/0000083-000

Kanter, J., Busch, A., \& Rusch, L. (2009). Behavioral activation. Abingdon: Routledge.

Leontiev, D. A. (2013a). Personal meaning: A challenge for psychology. Journal of Positive Psychology, 8(6), 459-470. doi: 10.1080/17439760.2013.830767

Leontiev, D. A. (2013b). Positive psychology in search for meaning: An introduction. Journalof Positive Psychology, 8(6), 457-458. doi: 10.1080/17439760.2013.830766

Maddi, S. R. (1970). The search for meaning. In M. Page(Ed.), The Nebraska Symposium on motivation (pp. 134-183). Lincolin: University of Nebraska Press.

Martela, F., \& Steger, M. F. (2016). The three meanings of meaning in life: Distinguishing coherence, purpose, and significance. Journal of Positive Psychology, 11(5), 531-545. doi: 10.1080/17439760.2015.1137623

Maslow, A. H. (2011). Introdução à psicologia do ser. São Paulo, SP: Martins Fontes. (Trabalho original publicado em 1962).

Mcknight, P., \& Kashdan, T. (2009). Purpose in life as a system that creates and sustains health and well-being: An integrative, testable theory. Review of General Psychology, 13(3), 242-251. doi: 10.1037/a0017152

Park, C. L. (2010). Making sense of the meaning literature: An integrative review of meaning making and its effects on adjustment to stressful life events. Psychological Bulletin, 136(2), 257-301. doi: 10.1037/a0018301

Park, C. L. (2013). The meaning making model: A framework for understanding meaning, spirituality, and stress-related growth in health psychology. European Health Psychologist, 15(2), 40-47. Recuperado de https://bit.ly/3eIWis6

Park, C., \& Folkman, S. (1997). Meaning in the context of stress and coping. Review of General Psychology, 1(2), 115-144. doi: 10.1037/1089-2680.1.2.115

Proulx, T. (2013). Meaning maintenance model: Introducing soren to existential social psychology. In J. A. Hicks \& C. Routledge (Orgs.), The experience of meaning in life: Classical perspectives, emerging themes, and controversies (pp. 47-59). Dordrecht: Springer. doi: 10.1007/978-94-007-6527-6_4

Proulx, T., \& Heine, S. J.(2008). The case of the transmogrifying experimenter: Affirmation of a moral schema following implicit change detection. Psychological Science, 19(12), 1294-1300. doi: 10.1111/j.1467-9280.2008.02238.x

Proulx, T., \& Inzlicht, M. (2012). The five "A"s of meaning maintenance: Finding meaning in the theories of sense-making. Psychological Inquiry, 23(4), 317-335. doi: 10.1080/1047840X.2012.702372

Rabin, A. I. (1979). Meaning and Void-Inner Experience and Incentives in People's Lives (Book). Journal of Personality Assessment, 43(2), 191. doi: 10.1207/s15327752jpa4302_20

Reker, G. T., \& Wong, P. T. P. (1988). Aging as an individual process: Toward a theory of personal meaning. In Emergent theories of aging (pp. 214-246). Springer.

Sartre, J.-P. (2014). O existencialismo é um humanismo. Petrópolis, RJ: Vozes. (Trabalho original publicado em 1946).

Seligman, M. E. (2019). Florescer: Uma nova compreensão da felicidade e do bem-estar. Rio de Janeiro, RJ: Objetiva.

Shin, J. Y., \& Steger, M. F. (2014). Promoting meaning and purpose in life. In A. C. Parks \& S. M. Schueller (Eds.), The Wiley Blackwell handbook of positive psychological interventions (pp. 90-110). Hoboken: Wiley-Blackwell. doi: 10.1002/9781118315927.ch5

Solomon, S., Greenberg, J., \& Pyszczynski, T. (1991). A terror management theory of social behavior: The psychological functions of self-esteem and cultural worldviews. Advances in Experimental Social Psychology, 24, 93-159. doi: 10.1016/S0065-2601(08)60328-7

Steger, M. F., Frazier, P., Oishi, S., \& Kaler, M. (2006). The meaning in life questionnaire: Assessing the presence of and search for meaning in life. Journal of Counseling Psychology, 53(1), 80-93. doi: 10.1037/0022-0167.53.1.80

Steger, M. F., Kawabata, Y., Shimai, S., \& Otake, K. (2008). The meaningful life in Japan and the United States: Levels and correlates of meaning in life. Journal of Research in Personality, 42(3), 660-678. doi: 10.1016/j.jrp.2007.09.003

Steger, M. F. (2012). Making Meaning in Life. Psychological Inquiry, 23(4), 381-385. doi: 10.1080/ 1047840X.2012.720832

Thir, M., \& Batthyány, A. (2016). The state of empirical research on Logotherapy and existential analysis. In A. Batthyány (Ed.), Logotherapy and existential analysis (pp. 53-74). Cham: Springer. 